\title{
Salas de Situação: Implantação de monitoramento inteligente como suporte às Políticas Públicas
}

\author{
Angelina da Silva Inácio, Adriano Galindo Leal, Vagner Luiz Gava, Alessandro \\ Santiago dos Santos
}

Unidade de Tecnologias Digitais - Instituto de Pesquisas Tecnológicas, Av. Prof. Almeida Prado, 532 - Butantã, São Paulo - SP, 05508-901 - Brasil

\{angelinasi, leal, vlgava, alesan\} @ipt.br

\begin{abstract}
This Organizational Report discusses the steps of data curation that are the basis of the Information and Intelligent Monitoring System (SIMI). SIMI is the main component of the Situation Room, created at the headquarters of the Government of the State of São Paulo, to monitor the evolution of the COVID-19 pandemic in the State. This Situation Room can provide, virtually through the Internet, epidemiological and hospital information, social isolation indices, electronic invoices issued, and other relevant data on the economy. All this information is anonymized and provided by public institutions and private companies. From the analysis provided by SIMI, it is possible to assess the population's adherence to the measures taken by the Government, the impacts on the spread of the disease, and the economic performance of the State.
\end{abstract}

Resumo. Este Relato Organizacional discorre sobre as etapas de curadoria de dados que consiste na base do Sistema de Informações e Monitoramento Inteligente (SIMI). O SIMI é o principal componente da Sala de Situação, criada na sede do Governo do Estado de São Paulo, para acompanhar a evolução da pandemia de COVID-19 no estado. Essa Sala de Situação pode fornecer, virtualmente através da Internet, informações epidemiológicas e hospitalares, indices de isolamento social, notas fiscais eletrônicas emitidas, dados sobre a economia, entre outras. Todas essas informações são anonimizadas e fornecidas por instituições públicas e empresas privadas. A partir das análises disponibilizadas pelo SIMI, pode-se avaliar a adesão da população às medidas tomadas pelo Governo, os impactos na disseminação da doença e no desempenho econômico do Estado.

\section{Introdução}

Derivando dos conceitos de [BUENO 2003], podemos definir que uma Sala de Situação do Século XXI é composta por uma infraestrutura de hardware, software e conectividade, bem como pessoal qualificado, para coordenar a operação durante o monitoramento de crises ou condições especiais. Sendo fundamental que possua capacidade de comunicação para receber informações em tempo real para apoiar um processo expedito de tomada de decisão como reação ao problema-alvo. Helvécio Bueno explica em [Bueno 2003] que originalmente, esse conceito deriva da expressão "sala de guerra", de origem militar, e sua função vem da necessidade em se monitorar situações de perigo eminente. Adicionalmente, Bueno cita Carlos Matus, economista 
chileno, que propõe que salas de situação também poderiam auxiliar no planejamento e monitoramento de ações governamentais.

A partir do início da Pandemia do COVID-19 (Corona Virus Disease 2019 SARS-Cov-2) decretada pela OMS em março de 2020 [OPAS/OMS Brasil 2020], várias salas de situação foram criadas ao redor do mundo para gerenciar a crise com estratégias para minimizar os impactos econômicos e evitar o colapso do sistema de saúde. E devido à essa emergência, todos os stakeholders têm interesse em colaborar no fornecimento de informações.

No Estado de São Paulo iniciou-se um compartilhamento de dados entre várias instituições públicas e empresas, em conformidade com os aspectos legais da Lei Geral De Proteção De Dados (LGPD), uma vez que apenas dados anonimizados e agrupados foram compartilhados. Após a Pandemia, provavelmente, esse compartilhamento deverá ser aperfeiçoado e incorporado à operação rotineira das instituições de forma a viabilizar respostas mais rápidas e eficientes numa próxima emergência, bem como aprimorar o gerenciamento do sistema de saúde, ou mesmo ser aplicado em outras áreas do governo, como transporte e desenvolvimento econômico.

O objetivo deste trabalho é descrever a estratégia global para as fontes de dados utilizadas com vistas ao monitoramento inteligente da pandemia em nível estadual. Apresentamos na Seção 2 a visão geral sobre a instituição do Sistema de Informações e Monitoramento Inteligente e na Seção 3, as ações realizadas para a criação da Sala de Situação. Na Seção 4, discorremos sobre alguns relatórios e painéis resultantes da curadoria de dados para monitoramento. Na Seção 5, apresentamos nossas considerações finais.

\section{Contexto}

Em maio de 2020, o Governo do Estado de São Paulo instituiu o SIMI (Sistema de Informações e Monitoramento Inteligente) através do Decreto No. 64.963 [Governo do Estado de São Paulo (GESP) 2020]. O Comitê Gestor do SIMI é composto por representantes da Administração Pública Estadual (Secretarias de Governo, Desenvolvimento Econômico e Saúde; e Instituto de Pesquisas Tecnológicas do Estado de São Paulo - IPT).

O SIMI destina-se a apoiar a formulação e avaliação das ações do Estado para a contenção da disseminação da COVID-19, acompanhamento dos índices de isolamento social e recuperação da atividade econômica. Para tanto, são coletados, de forma anonimizada, dados epidemiológicos, dados sociais do IBGE e SEADE, taxa de ocupação de leitos nos hospitais e emissão de notas fiscais eletrônicas. Dentre as suas atribuições incluem: realizar a curadoria de dados e informações públicas com relação à evolução da pandemia e seu impacto sobre o sistema de saúde; elaborar relatórios e analisar cenários de progressão da disseminação pela COVID-19 de acordo com essas informações; consolidar parcerias que contribuam para a coleta e análise das informações.

\section{Ações Realizadas}

Para a implantação da Sala de Situação, escolheu-se o espaço físico do Palácio dos Bandeirantes, sede do Governo do Estado de São Paulo, e os recursos necessários: servidores, softwares, telões, mobiliário, instalações elétricas e de Internet. As equipes 
constituintes da Sala de Situação foram definidas de acordo com suas competências e das principais demandas a serem atendidas. Grupos multidisciplinares são citados como fatores de sucesso em iniciativas de Dados Abertos, como no trabalho de Iryna Susha [Susha et al. 2015].

Neste trabalho, o foco inicial foram informações sobre a progressão da pandemia, as previsões epidemiológicas (casos confirmados, óbitos, média móvel) e a adesão à quarentena decretada pelo Governo Estadual, dada pelos índices de isolamento social. Posteriormente, foram agregadas outras fontes de dados necessárias e celebraram-se- acordos entre entidades públicas e privadas para seu uso:

- Dados epidemiológicos: são coletados de sites oficiais de outros países e do Ministério da Saúde do Brasil. As previsões de casos e óbitos são calculadas por Pesquisadores Estatísticos;

- Índices de isolamento social: obtidos através de um acordo entre Governo Estadual, operadoras de telefonia e Associação Brasileira de Recursos em Telecomunicações;

- Taxa de ocupação de leitos de hospitais: fornecidos pela Secretaria de Estado de Saúde;

- Recuperação Econômica: dados de Notas Fiscais e Cupons Fiscais Eletrônicos emitidos pelas empresas e encaminhados à Secretaria Estadual da Fazenda.

Importante destacar que havia uma equipe dedicada à coleta e curadoria (tratamento) dos dados, dividida por temática, para que estes pudessem ser analisados de forma holística. Ao serem coletados, os dados passam por um processo de ETL (Extract, Transform, Load) e são importados em ferramentas de Business Intelligence para criar as visualizações dos dados. Dashboards (painéis com gráficos e indicadores) e boletins epidemiológicos são disponibilizados em páginas oficiais do governo e em salas de situação em que os seus integrantes (pesquisadores, consultores, assessores, secretários e outros membros do poder público), imprensa e público em geral poderão consultá-los e usá-los em suas análises. Os dashboards são exibidos de forma constante nessa sala em telões em alta definição (videowall). Para manter a privacidade dos cidadãos e empresas, todos os dados são anonimizados e agrupados por data, país, estado, município e regiões de interesse.

\section{Resultados}

\subsection{Isolamento social}

Também conhecido como "distanciamento social", como explicado em [UFRGS 2020], o isolamento social busca diminuir a interação social entre pessoas para reduzir a velocidade de transmissão do vírus quando há transmissão comunitária (quando não é possível rastrear a origem do contágio, ou seja, o vírus já está circulando livremente entre a população). O Governo Estadual, ao decretar a quarenta, buscou aplicar medidas de isolamento onde apenas serviços essenciais (hospitais, farmácias, supermercados etc.) devem funcionar e demais setores da indústria, comércio e serviços são temporariamente fechados. Atividades que causem aglomerações devem ser evitadas ocorre o fechamento de escolas, academias, recomenda-se o teletrabalho, quando possível e cancelamento de eventos públicos. 
Por meio dessas medidas para reduzir o crescimento do contágio, promove-se mecanismos para gerir de maneira efetiva insumos médicos, leitos de enfermaria e UTI, respiradores, profissionais e evitar o colapso do sistema de saúde [Ministério da Saúde 2021]. A partir dos índices de isolamento podem-se analisar os padrões de mobilidade da população e adesão às medidas de restrição de circulação.

Conforme informado em [Conexis 2020], em maio de 2020, foi estabelecida a parceria entre as principais prestadoras de serviços de telecomunicações e governos estaduais e municipais para o uso da plataforma de Big Data gerida pela Associação Brasileira de Recursos em Telecomunicações - ABR TELECOM. Na ocasião do acordo foram disponibilizados nesta plataforma os índices de isolamento para 12 estados e 14 municípios brasileiros.

Para o cálculo do índice de isolamento, descrito por [Instituto de Pesquisas Técnológicas (IPT) 2020], as operadoras de telefonia baseiam-se na localização dos celulares conectados às antenas (ERBs - Estações Rádio Base). Considera-se como domicílio do celular conectado à antena aquele em que ele se encontra das $22 \mathrm{~h} 00$ às 2h00. Caso o celular se conecte em outra ERB, diferente da ERB de seu local de residência durante o dia, ele é considerado fora do índice de isolamento.

Os índices são atualizados diariamente [Instituto de Pesquisas Técnológicas (IPT) 2020], porém com os valores referentes ao dia anterior. Essa frequência é necessária para que as operadoras façam o processamento dos índices, que consiste em agregar os dados recebidos e torná-los anônimos para assegurar a privacidade de seus usuários (Figura 1). Os governos não têm acesso a quaisquer dados pessoais relacionados aos celulares avaliados.

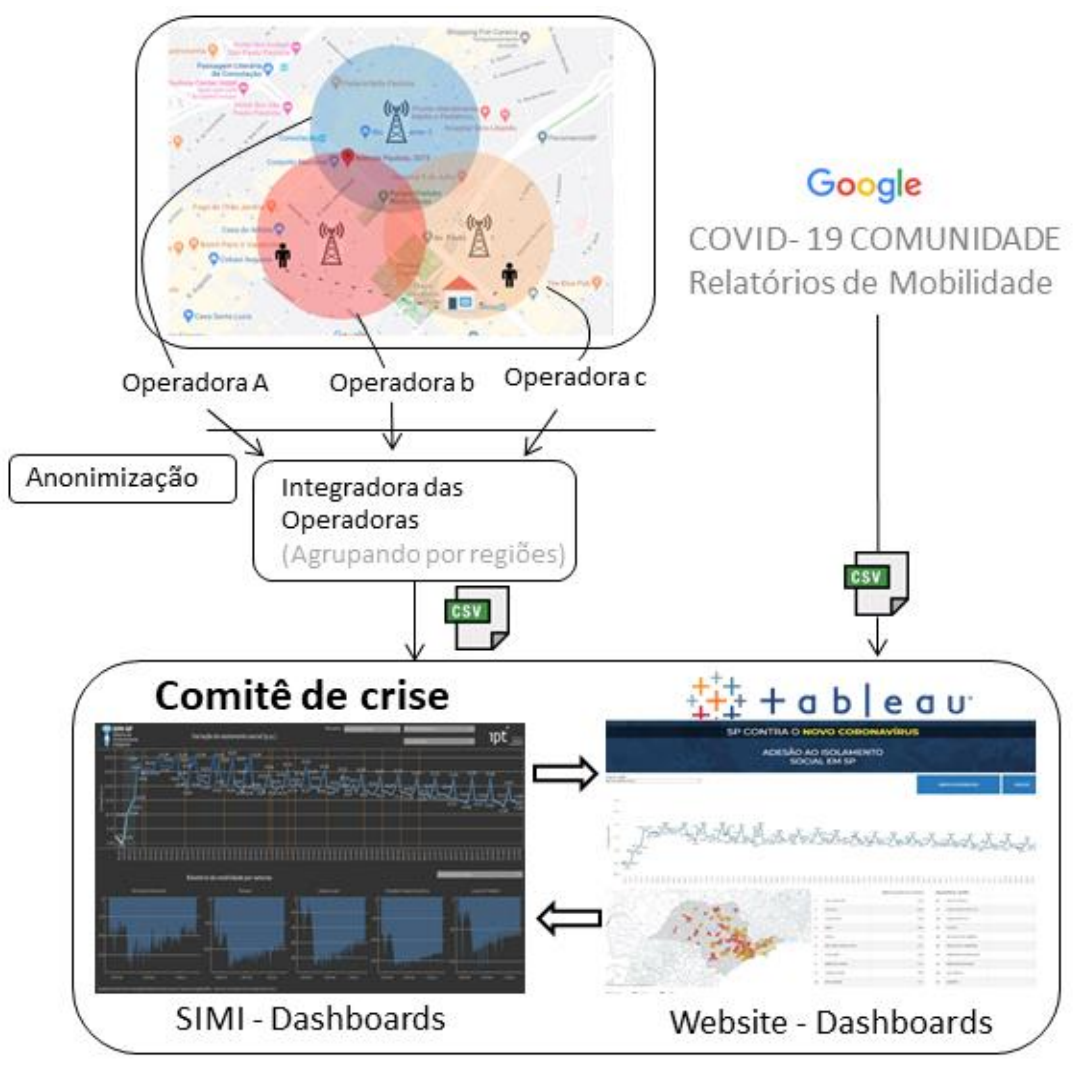

Figura 1. Coleta de dados das operadoras [Santos et al. 2021] 
Exemplos de dashboards sobre isolamento social disponibilizados na sala de situação e sites das entidades públicas:

- Índice de adesão ao isolamento social: Painel contendo gráfico com o valor do índice por data, com início em fevereiro/2020. Podem-se visualizar os dados por município ou a média do Estado. Há uma versão alternativa que inclui gráficos com os dados do Relatório de Mobilidade da empresa Google ${ }^{1}$;

- Mapa do Estado: Painel contendo gráfico geolocalizado do Estado, com os níveis de isolamento de cada município representados por escala de cores;

- Ranking absoluto e relativo: Painel com os municípios com maior índice de isolamento, classificados em ordem decrescente, considerando dados absolutos ou relativos (valor de acréscimo em relação aos índices antes da pandemia);

- Dados: Tabela dos municípios disponibilizados na plataforma de Big Data, com seus dados de população e índice de isolamento por data.

\subsection{Dados epidemiológicos e ocupação de leitos}

Para demonstrar a situação atual da pandemia, números de casos e óbitos são disponibilizados pela Fundação Sistema Estadual de Análise de Dados (SEADE), que coleta e unifica os dados de outras instituições vinculadas à Secretaria de Saúde do Estado (para municípios e do Estado) e do Conselho Nacional de Secretários de Saúde (CONASS, para informações sobre o Brasil) [SEADE 2021]. Outros países de interesse também são incluídos e são consultados a partir de portais oficiais governamentais ou de instituições públicas de ensino e pesquisa, como o Johns Hopkins Coronavirus Resource Center. Os números correspondem ao dia anterior.

Os dados de casos e óbitos também são utilizados por uma equipe de pesquisadores especializados em Estatística para criar projeções de cenários possíveis da evolução da pandemia.

A capacidade do sistema de saúde é avaliada com base na média móvel de 7 dias de leitos de UTI e Enfermaria destinados à pacientes suspeitos ou confirmados com COVID-19 [SEADE 2021]. A taxa de ocupação é calculada a partir de números fornecidos pela Secretaria de Saúde para a Instituição Pública.

Além de arquivos em texto (formato CSV) e planilhas, disponíveis no portal do Governo, as informações são exibidas em gráficos de linhas, com escala linear ou logarítmica, onde é possível selecionar as regiões geográficas de interesse para visualização individual ou múltiplas regiões para compará-las entre si.

\subsection{Informações sobre a Recuperação Econômica}

Conforme discutido por [Schuchmann et al. 2020], mesmo sem as medidas de isolamento social, indústrias, comércio e serviços podem sofrer reveses causados pela "queda acentuada e persistente da atividade econômica".

\footnotetext{
${ }^{1}$ https://www.google.com.br/covid19/mobility/
} 
Para auxiliar a análise da atual situação econômica do Estado e sua retomada fomentada por um plano de recuperação da economia, o Plano São Paulo ${ }^{2}$, são usados dados de Notas Fiscais e Cupons Fiscais Eletrônicos (documentos digitais que registram arquivos e operações gerados por contribuintes credenciados pela Secretaria da Fazenda e pelo comércio varejista). Esses valores são gerados de acordo com as regras de privacidade e sigilo fiscal, determinadas pelos Artigos 198 e 199 do Código Tributário Brasileiro, Resolução Interna SF 20 da Secretaria Estadual da Fazenda, e Artigo 325 do Código Penal Brasileiro, para garantir a impossibilidade de identificação do contribuinte [Santos et al. 2021]. A Figura 2 exemplifica o processo para as notas fiscais eletrônicas. A Sala de Situação recebe atualizações semanais da Secretaria.

Os valores dos documentos físcais são agrupados de acordo com data, localização do ponto de venda (apenas consideram-se Estado e Regional de Saúde) e setores da economia, sendo estes Atacado, Combustíveis, Industria e Varejo e subsetores dos supracitados. Os valores de receitas são aproximados pois não é possível verificar ocorrências como retornos, remessas ou transferências. [Santos et al. 2021]

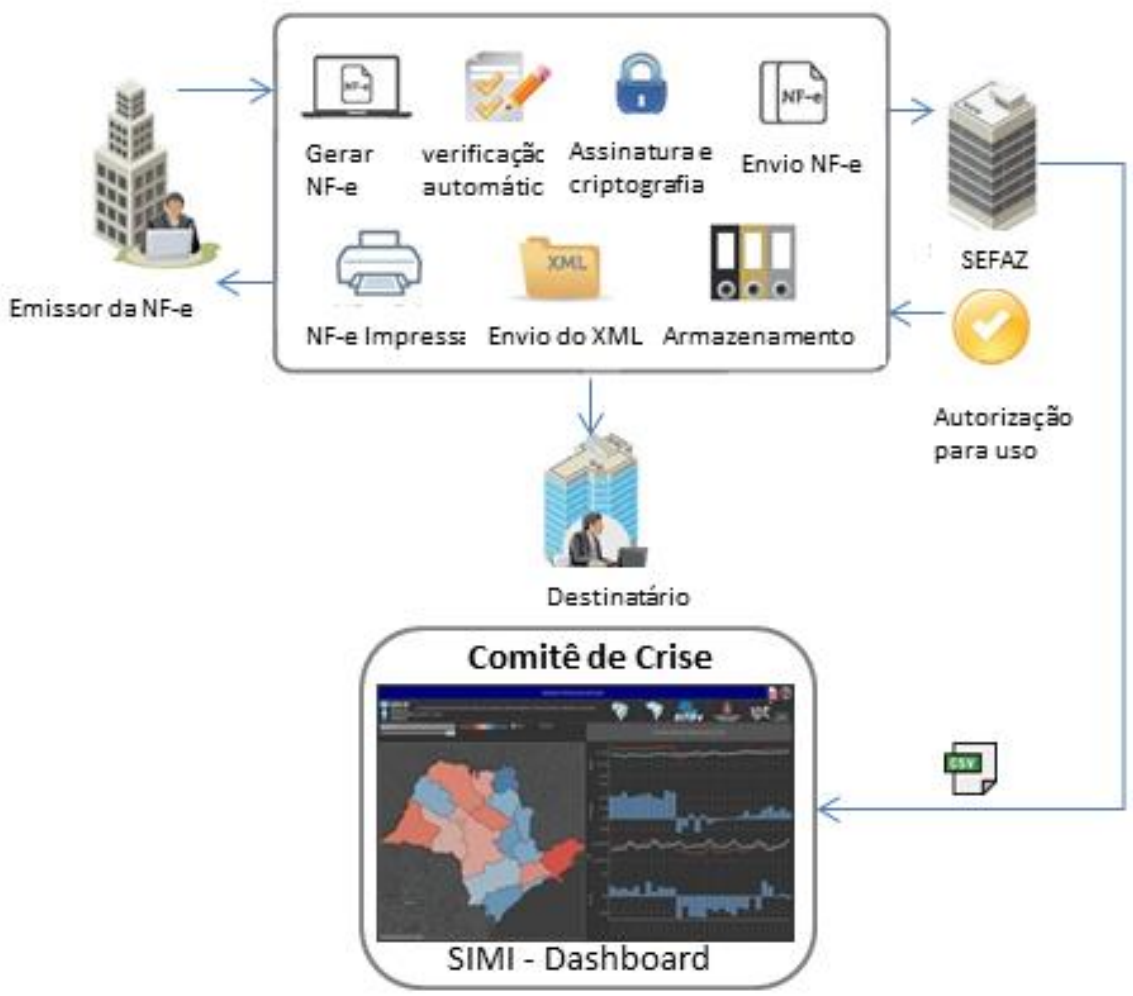

Figura 2. Processo de coleta dos dados [Santos et al. 2021]

Os dashboards sobre os números da economia são exibidos na Sala de Situação. Alguns exemplos de painéis criados:

- Consumo Intermediário: Painel com gráficos sobre vendas para pessoas jurídicas, com valores, quantidade e variação percentual entre os anos

\footnotetext{
${ }^{2}$ https://www.saopaulo.sp.gov.br/planosp/
} 
selecionados (2020 - 2019, 2021 - 2020, 2021 - 2019) e filtro por Regional de Saúde (Departamentos Regionais de Saúde - DRS);

- Consumo Final: Idem ao anterior, porém considerando vendas para pessoas físicas;

- Estado: Além dos gráficos com os valores de vendas, há um mapa do estado com escala de cores de acordo com a variação percentual entre os anos. Pode-se acompanhar a evolução dos números ao decorrer das semanas dos anos selecionados;

- Setores / Subsetores: Contém gráficos com a mesma estrutura dos anteriores (Consumo Intermediário e Final), com valores das vendas por setores econômicos e seleção por setor ou subsetor. Para Setores, há filtro por Regional de Saúde.

\section{Lições aprendidas}

Um desafio típico desse tipo de projeto é a disposição para o compartilhamento de dados entre os diversos "silos de informação" nas entidades públicas e privadas. Todavia, devido a premência da Pandemia da COVID-19, as resistências naturais foram quebradas e testemunhou-se grande e inédita cooperação entre as diversas esferas governamentais e as empresas do setor público e privado para a criação de indicadores relevantes para direcionar os esforços emergenciais e criar uma plataforma de divulgação dessas informações. Dada a excepcionalidade da situação, problemas típicos como compras de hardware, software e contratação de pessoas foram resolvidos através de doações de materiais por empresas privadas e disponibilização de funcionários competentes do IPT e de outros Órgãos Governamentais.

Um aspecto que creditamos como o fator primordial para o sucesso e disponibilização dos dados pelas diversas entidades, foi a formalização através de Decreto Governamental do Grupo Gestor dessa Sala de Situação. Esse formalismo combinado com a formação de grupos multidisciplinares foram os principais fatores para estar funcionando de forma adequada nos últimos 15 meses e sem data para o encerramento das atividades ou da Pandemia.

A criação da Sala de Situação, um ambiente físico combinado com um portal virtual, com informações relevantes que podem ser consultadas por todos, oferece transparência sobre as ações do poder público e aumentam a confiança da sociedade. Os dados abertos e disponíveis também tiveram um caráter educativo que manteve o engajamento da população às medidas de higiene e isolamento social, viabilizando que os serviços de saúde conseguissem atender às demandas. $\mathrm{O}$ caráter educativo, além das informações técnicas disponibilizadas, veio na forma da população aprender que medidas mais restritivas reduziam internações e mortes, enquanto datas festivas de maior circulação de pessoas pioravam os indicadores.

Finalmente, espera-se que perdure o comportamento de compartilhamento de dados anonimizados, em conformidade com os aspectos legais da LGPD. Preferencialmente, que seja estabelecido através de novos Decretos, para que outras emergências possam ser detectadas precocemente e controladas em tempo real, uma vez que esse processo estará incorporado às operações rotineiras das instituições. 


\section{Agradecimentos}

Os autores desejam agradecer ao suporte recebido através dos processos $\mathrm{n}^{\circ}$ \#2019/01664-6 e \#2017/50343-2, Fundação de Amparo à Pesquisa do Estado de São Paulo (FAPESP).

\section{Referências}

Bueno, H. (2003). "Utilização da Sala de Situação de Saúde no Distrito Federal". Universidade de Brasília.

Conexis (2020). "12 estados e 14 prefeituras já usam a plataforma das operadoras para identificar concentrações”. https://conexis.org.br/12-estados-e-14-prefeituras-jausam-a-plataforma-das-operadoras-para-identificar-concentracoes/, acessado em 13 de abril de 2021.

Governo do Estado de São Paulo (GESP) (2020). "Decreto n ${ }^{0}$ 64.963, de 05 de Maio de 2020." . Diário Oficial do Estado de São Paulo. https://www.al.sp.gov.br/repositorio/legislacao/decreto/2020/decreto-6496305.05.2020.html, acessado em 11 de abril de 2021.

Instituto de Pesquisas Técnológicas (IPT) (2020). "Perguntas sobre isolamento social". IPT. https://www.ipt.br/noticia/1623-_perguntas_sobre_isolamento_social.htm, acessado em 7 de abril de 2021.

Ministério da Saúde (2021). "Medidas não farmacológicas". https://coronavirus.saude.gov.br/medidas-nao-farmacologicas, acessado em 7 de abril de 2021.

OPAS/OMS Brasil (2020). "OMS afirma que COVID-19 é agora caracterizada como pandemia". https://www.paho.org/pt/news/11-3-2020-who-characterizes-covid-19pandemic, acessado em 7 de abril de 2021.

Santos, A. S., Teixeira, I. C., Neves, R., et al. (2021). "Challenges and Strategies for Information Systems in the Decision-Making Process to Face the COVID-19 Pandemic: The São Paulo Case". In Trends and Applications in Information Systems and Technologies. p. 630-640.

Schuchmann, A. Z., Schnorrenberger, B. L., Chiquetti, M. E., et al. (2020). "Isolamento social vertical $\mathrm{X}$ Isolamento social horizontal: os dilemas sanitários e sociais no enfrentamento da pandemia de COVID-19”. Brazilian Journal of Health Review, v. 3, n. 2, p. 3556-3576.

SEADE (2021). "Notas, variáveis, fontes de dados e agregações territoriais". https://www.seade.gov.br/wp-content/uploads/2021/04/Boletim-Coronavirus-Anexometodologico.pdf, acessado em 14 de abril de 2021.

Susha, I., Zuiderwijk, A., Charalabidis, Y., Parycek, P. e Janssen, M. (2015). "Critical factors for open data publication and use: A comparison of city-level, regional, and transnational cases". eJournal of eDemocracy and Open Government, v. 7, n. 2, p. 94-115.

UFRGS (2020). "Qual a diferença de distanciamento social, isolamento e quarentena?" https://www.ufrgs.br/telessauders/posts_coronavirus/qual-a-diferenca-dedistanciamento-social-isolamento-e-quarentena/, acessado em 7 de abril de 2021. 